\title{
FORMATION OF BOXY/PEANUT-SHAPED BULGES IN SPIRAL GALAXIES: ACCRETION OR BAR INSTABILITY?
}

\author{
M. BUREAU AND K.C. FREEMAN \\ Mount Stromlo and Siding Spring Observatories \\ Private Bag, Weston Creek P.O., ACT 2611, Australia
}

\section{Introduction}

Boxy/peanut-shaped bulge galaxies constitute at least $20-30 \%$ of all spirals. Distribution functions and numerical simulations studies have shown that the slow decay of the orbit of a companion into a larger spiral can lead to the formation of a boxy/peanut bulge. However, the bar-buckling instability now appears a more likely process. Thin bars either do not form or, as shown by N-body simulations, buckle and settle with an increased thickness, appearing boxy or peanut-shaped when seen edge-on. This project aims to determine the dynamical state of a sample of 30 edge-on spirals, $3 / 4$ of which have boxy/peanut-shaped bulges, half having close-by companions.

\section{Observations and Discussion}

We have obtained high $\mathrm{S} / \mathrm{N}$ long-slit spectroscopy along the major axis of all our sample galaxies. Recent or ongoing accretion events are detectable as irregularities in the position-velocity diagrams (PVDs) obtained, while a double-peaked PVD denotes the presence of a bar. Of the 16 boxy/peanut galaxies with extended emission lines, 11 display a clear bar signature. Those galaxies also possess bulge morphologies resembling those of $\mathrm{N}$-body simulations. Only 2 galaxies (with extreme bulge morphologies) show signs of accretion, and 3 have regular PVDs but are also very dusty. Furthermore, none of the galaxies with a normal spheroidal bulge show signs of a bar.

Our results point to a picture where most boxy/peanut-shaped bulges are due to the presence of a thick bar seen edge-on, and only a few extreme cases are formed through accretion. The possibility of hybrid scenarios where a bar is excited by an encounter and then buckles remains. 